\title{
Influence of Atomizing Gas and Cooling Rate on Solidification Characterization of Nickel-based Superalloy Powders
}

\author{
Fang Pengjun ${ }^{1}, \quad \mathrm{Xu} \mathrm{Yi}^{1,2}, \quad$ Li Xinggang $^{3}, \quad{\text { Chen } \mathrm{Ya}^{4}}^{4}$ \\ ${ }^{1}$ School of Materials Science \& Engineering, Southwest Jiaotong University, Chengdu 610031, China; ${ }^{2}$ Foundation Institute of Materials \\ Science, Bremen University, Bremen 28359, Germany; ${ }^{3}$ National Engineering \& Technology Research Center for Nonferrous Metal Matrix \\ Composites, General Research Institute for Nonferrous Metals, Beijing 100088, China; ${ }^{4}$ Research Institute of Physical Culture, Southwest \\ Jiaotong University, Chengdu 610031, China
}

\begin{abstract}
Nickel-based superalloy powders have been produced by high pressure argon gas and nitrogen gas atomization, separately. The microstructural characterization of nickel-based alloy powders has been performed by a scanning electron microscope equipped with an EDS microanalysis unit. Based on a Newtonian cooling model, the flight speed and the cooling rate of two kinds of alloy droplets were calculated. The results show that the droplet cooling rate, which depends on atomization medium and droplet size, has an effect on the solidification microstructure. For argon-gas atomized powders, the developed dendrite structure is predominant at a lower cooling rate and a mixed microstructure composed of dendrite structure and cellular structure is observed at a higher cooling rate. For nitrogen-gas atomized powders, the dendrite structure is predominant at a lower cooling rate and a fullcellular str ucture can be observed at a higher cooling rate. According to calculation, the cooling rate of argon-gas atomized droplets is in a range from $1.0 \times 10^{5} \mathrm{~K} \cdot \mathrm{s}^{-1}$ to $4.24 \times 10^{6} \mathrm{~K} \cdot \mathrm{s}^{-1}$, while the cooling rate of nitrogen-gas atomized droplets is from $1.0 \times 10^{5} \mathrm{~K} \cdot \mathrm{s}^{-1}$ to $4.8 \times 10^{6} \mathrm{~K} \cdot \mathrm{s}^{-1}$. The cooling rate increases with decreasing of droplets diameter. Two kinds of atomizing gases have a slight influence on the cooling rate of droplets. The dendrite axis is rich in elements such as $\mathrm{Cr}, \mathrm{Co}, \mathrm{W}, \mathrm{Ni}$ and $\mathrm{Al}$ while the inter-dendrite region is rich in Ti element.
\end{abstract}

Key words: nickel-based superalloy powders; gas atomization; solidification microstructure; cooling rate

Nickel-based alloy powders produced by gas atomization process are key raw materials for the fabrication of critical aircraft structural components subject to high temperatures. The nickel-based powder metallurgy (PM) superalloy possesses a high volume fraction of $\gamma^{\prime}$-strengthening phase and shows excellent mechanical properties at an operation temperature above $650{ }^{\circ} \mathrm{C}$, such as chemical stability properties, high corrosion resistance, no macro-segregation, and resistance to thermal fatigue and thermal shock ${ }^{[1-5]}$. It is also an ideal material for jet engines or gas turbine engines of the advanced aero-engine. Gas atomization is a preferred method for powders production. During the gas-atomization process, a liquid stream is typically disintegrated into a large number of various sized droplets by high-speed gas jets. As all of the solid state materials such as minerals, ceramics and metals may be converted into the liquid state by melting, and powders from all of these materials may be produced via atomization of the liquid melt followed by a rapid solidification of the droplets in the spray chamber ${ }^{[6]}$. The powders have outstanding properties which cannot be obtained by conventional processing routes. Commonly, owing to the very high cooling rate achieved in the atomization process, there are decreased segregation, increased solid solubility, new metastable phases and a finer microstructure of powders or of consolidated products ${ }^{[7-9]}$. The properties of gas-atomized powders are strongly related

Received date: February 14, 2017

Foundation item: National Natural Science Foundation of China (51301143); the National Postdoctoral Foundation of China (2014M560727); the Sichuan Province Science-Technology Support Plan, China (2015GZ0228); the Science and Technology Innovation Project of SWJTU University, China (2682014CX001)

Corresponding author: Xu Yi, Ph. D., Associate Professor, School of Materials Science \& Engineering, Southwest Jiaotong University, Chengdu 610031, P. R. China, Tel: 0086-28-87600782, E-mail: xybwbj@swjtu.cn

Copyright (C) 2018, Northwest Institute for Nonferrous Metal Research. Published by Elsevier BV. All rights reserved. 
to particle size and particle shape, which in turn are believed to have an apparent effect on the mechanical performance. The microstructure evolution is also important because it provides valuable information on particle size and droplet cooling rate, for example, the secondary dendrite arm spacing is closely associated with particle size and droplet cooling rate. The powder microstructures are of fundamental importance to optimize the atomization process parameters. A widespread research on the gas-atomization of various alloys has been performed, such as $\mathrm{Cu}-\mathrm{Sn}^{[10]}, \mathrm{Al}-\mathrm{Cu}$ alloy ${ }^{[11]}, \mathrm{Al}-\mathrm{Fe}^{[12]}$ and $\mathrm{Fe}-\mathrm{Ni}$ alloy $^{[13]}$. However, at present, there are few studies focusing on the effect of different atomization media on the powders microstructure and the cooling rate of nickel-based superalloy system. Up to date, it is still a great challenge to measure the cooling rates of flying droplets in a spray chamber by a measuring instrument. Thus, the aim of this paper is to study the effect of different atomization media like argon gas and nitrogen gas on powders microstructure, flight speed, cooling rate and micro-segregation of nickel-based alloy powders. This is accomplished through a series of experimental investigations involving by use of scanning electron microscope (SEM) and energy dispersive spectrometer (EDS). A Newtonian cooling model is employed to calculate the droplet cooling rate during the flight process. Thermal history calculation of atomized nickel-based alloy droplets and microstructure characterization of the final alloy powders performed. The present work is helpful to optimize the atomization process parameters for the production of nickel-based alloy powders.

\section{Experiment}

Nickel-based superalloy powders have been obtained through argon-gas atomization and nitrogen-gas atomization, separately. The detailed chemical composition $(w t \%)$ is listed in Table 1. The master alloy was melted in a magnesia crucible by induction heating and was superheated at $200 \mathrm{~K}$ to ensure a smooth flow of the melt. Atomization of the melt alloy was carried out under the melt-nozzle exit by argon gas at $3.9 \mathrm{MPa}$ and nitrogen gas at 3.6 MPa. Here, considering the fact that the pressure to yield of fine fraction of argon gas atomized powders was 3.8 3.9 $\mathrm{MPa}$, nitrogen gas atomized powders was about $3.6 \mathrm{MPa}$ according to our atomization process. For this reason, in this study, we did not choose the totally same pressure to conduct our experiment. Gas atomization usually produces a broad range of particles size. In the present work, the powders with a size range between $10 \mu \mathrm{m}$ and $200 \mu \mathrm{m}$ were selected for investigation. As the cooling rate of powders varied with particle size, a sieving procedure was applied in order to ensure the relationship between cooling rate, microstructure and powder particle size. Particles in the size ranges of $<44 \mu \mathrm{m}, 44 \sim 80 \mu \mathrm{m}$, $80 \sim 125 \mu \mathrm{m}$ and 125 200 $\mu \mathrm{m}$ were selected for further analysis. A certain amount of powders were taken into a beaker and anhydrous alcohol was added for ultrasonic cleaning. The detailed microstructures of the atomized powders were characterized by QUANTA200 scanning electron microscopy (SEM), and the dendrite segregation was analyzed with a Phoenix energy dispersive spectroscopy (EDS). The cooling rates and the flight speeds of atomized droplets were calculated by MATLAB10.0.

\section{Results and Discussion}

\subsection{Surfaces morphology of powder particles}

SEM observations of the powders atomized by argon gas are given in Fig.1 a and 1b. It can be observed that all of the powders are spherical and ellipsoidal in shape, and these powders have a good surface smoothness. Besides, several satellite powders adhere to some large powder particles surfaces (Fig.1b), which are induced by the collision of the droplets during the flight process in the spray chamber. Fig.1c and 1d show the surface morphology of nitrogen-gas atomized powders. Direct observations of nitrogen-gas

Table 1 Chemical composition of studied Nickel-based superalloy powders $(\mathbf{w t} \%)$

\begin{tabular}{ccccccccc}
\hline $\mathrm{Cr}$ & $\mathrm{W}$ & $\mathrm{Co}$ & $\mathrm{Mo}$ & $\mathrm{Nb}$ & $\mathrm{Al}$ & $\mathrm{C}$ & $\mathrm{Ti}$ & $\mathrm{Ni}$ \\
\hline 12.84 & 3.65 & 7.98 & 3.62 & 3.37 & 3.45 & 0.06 & 2.45 & Bal. \\
\hline
\end{tabular}

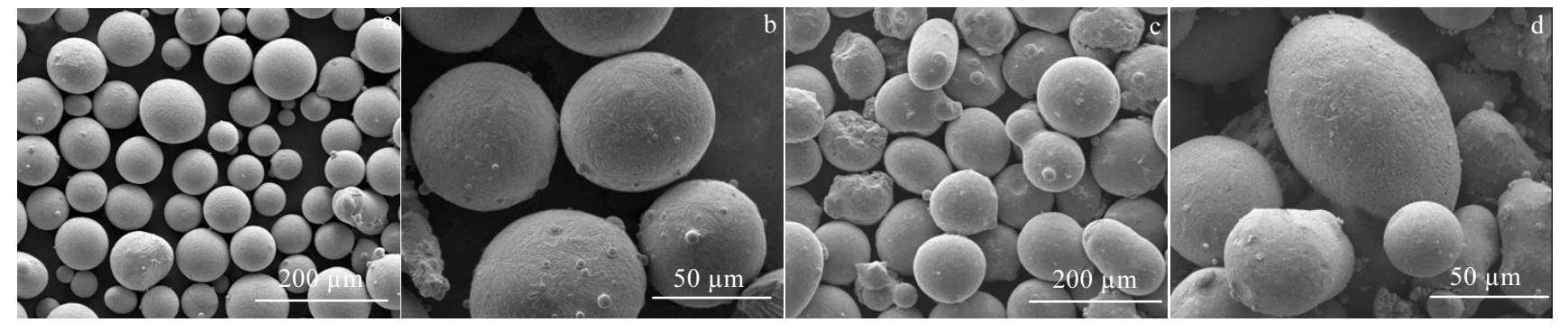

Fig. 1 Surface morphologies of the nickel-based alloy powder: $(a, b)$ argon-gas atomized powders; (c, d) nitrogen-gas atomized powder 
atomized particle surfaces show that several small powders are spherical in shape. Most particles, especially the large particles, are coarse and are not really spherical. Furthermore, a lot of small satellite particles stick on the surface of large particles. The comparison between two kinds of powders indicates that the appearance of the argon-gas atomized powders is much more regular and spherical than that of the nitrogen-gas atomized powders.

\subsection{Calculation of droplets flight speed and cooling rate}

The cooling rate of single alloy droplets depends on the gas flow velocity and flight distance during the atomization process, and the axial gas flow velocity is described as ${ }^{[14]}$.

$$
u=u_{0}\left[1+\left(\frac{x}{\lambda}\right)^{15}\right]^{-0.033}
$$

where $u$ and $u_{0}$ are the axial gas flow velocity and the initial axial gas flow velocity at the nozzle exit, respectively, $x$ is the axial distance, and $\lambda$ is a constant related to the attenuation of gas flow velocity in the axial direction which can be expressed as $\lambda=\alpha \sqrt{A_{\mathrm{e}}}$, where $\alpha$ is an empirical constant related to gas dynamics viscosity, taking $\alpha=10.5, A_{\mathrm{e}}$ is the nozzle exit area, taking $A_{\mathrm{e}}=\pi R_{0}^{2}$ and $R_{0}$ being the nozzle exit radius. The initial velocity $u_{0}$ can be written as ${ }^{[15]}$ :

$$
\begin{aligned}
& u_{0}=\frac{J_{\mathrm{g}}}{\rho_{\mathrm{g}} A_{\mathrm{e}}} \\
& J_{\mathrm{g}}=A_{t} \frac{P_{0}}{\sqrt{R T_{0}}} \sqrt{\gamma\left(\frac{2}{\gamma+1}\right)^{\frac{\gamma+1}{\gamma-1}}}
\end{aligned}
$$

where $J_{\mathrm{g}}$ is the gas flow, $\rho_{\mathrm{g}}$ is the gas density, $A_{\mathrm{t}}$ is the nozzle throat area, taking $A_{\mathrm{t}}=\pi R_{\mathrm{t}}^{2}, R_{\mathrm{t}}$ is the nozzle throat radius, $P_{0}$ is the pressure of atomizing gas in the atomizer (i.e. static pressure), $T_{0}$ is the gas temperature in the atomizer (i.e. static temperature) and $\gamma$ is the specific heat ratio. During the initial stage, metallic droplets are accelerated due to the action of the drag force of the atomizing gas stream. The role of the drag force on the acceleration of melting droplets can be expressed by the Newton's second law ${ }^{[16]}$ :

$$
\begin{aligned}
& \frac{\mathrm{d} v}{\mathrm{~d} t}=\frac{3}{4} \cdot \frac{C_{\mathrm{D}}}{d} \cdot \frac{\rho_{\mathrm{g}}}{\rho_{\mathrm{d}}}|u-v|(u-v)-g\left(1-\frac{\rho_{\mathrm{g}}}{\rho_{\mathrm{d}}}\right) \\
& \frac{\mathrm{d} v}{\mathrm{~d} t}=\frac{\mathrm{d} x}{\mathrm{~d} t} \cdot \frac{\mathrm{d} v}{\mathrm{~d} x}=v \cdot \frac{\mathrm{d} v}{\mathrm{~d} x}
\end{aligned}
$$

where $v$ is droplet velocity, $C_{\mathrm{D}}$ is drag coefficient, $d$ is droplet diameter, $\rho_{\mathrm{g}}$ is gas density, and $\rho_{\mathrm{d}}$ is droplet density. The drag coefficient can be expressed as ${ }^{[17]}$ :

$$
\begin{aligned}
& C_{\mathrm{D}}=0.28+\frac{6.0 \sqrt{R e}+21.0}{R e} \\
& R e=\frac{\rho_{\mathrm{g}}|u-v| d}{\mu_{\mathrm{g}}}
\end{aligned}
$$

$R e$ is droplet Reynolds number, $\mu_{\mathrm{g}}$ is gas dynamic viscosity, $d$ is droplet diameter and $|u-v|$ is the relative velocity between metallic droplets and gas flow. The thermophysical parameters of argon gas (Ar) and nitrogen gas $\left(\mathrm{N}_{2}\right)$ are listed in Table $2^{[18,19]}$. Some atomization process parameters, as well as properties of nickel-based superalloy used in calculation, are evaluated as follows, $R_{\mathrm{e}}=0.0014, R_{\mathrm{t}}=0.0012$, $\gamma=1.4, \quad T_{0}=300 \mathrm{~K}, \rho_{\mathrm{d}}=8280 \mathrm{~kg} / \mathrm{m}^{3}, \quad C_{\mathrm{l}}=434 \mathrm{~J} /(\mathrm{kg} \cdot \mathrm{K}), T_{\mathrm{d}}$ $=1573 \mathrm{~K}^{[20]}$. By Eqs. (1) (3), the atomization gas velocity along the axis can be obtained. By Eqs. (1) (7), the droplets flight speed can be calculated. The variations of droplets velocity along the flight distance are shown in Fig.2 and Fig.3, respectively.

Fig. 2 and Fig. 3 show the changes in droplet velocity with the axial distance of argon gas and nitrogen gas atomization, respectively, for nickel-based superalloy droplets of 5, 25, $45,65,85,105,125$ and $145 \mu \mathrm{m}$ in diameter. The gas velocity slows down gradually with the flight distance. Each metallic droplet is accelerated up to the maximum velocity where the droplet velocity becomes equal to the instantaneous velocity of atomizing gas, i.e. the slip velocity between droplet and gas is zero. The droplet size has a direct influence on the flight speed. The smaller droplets have a lower value of the Reynolds number and this implies stronger drag force for the smaller droplet because the drag coefficient is inversely proportional to the Reynolds number as seen from Eq.(6). Hence, the small droplets are more easily influenced by the gas-dynamic drag force, and the maximum velocity for the smaller droplet

Table 2 Some parameters about atomizing gas ${ }^{[18,19]}$

\begin{tabular}{ccc}
\hline Parameter & Argon gas (Ar) & Nitrogen gas $\left(\mathrm{N}_{2}\right)$ \\
\hline$C_{\mathrm{pg}} / \mathrm{J} \cdot \mathrm{kg}^{-1} \cdot \mathrm{K}^{-1}$ & 540 & 1050 \\
$\rho_{\mathrm{g}} / \mathrm{kg} \cdot \mathrm{m}^{-3}$ & 1.78 & 1.25 \\
$\mu_{\mathrm{g}} / \times 10^{-5} \mathrm{~kg} \cdot \mathrm{m}^{-1} \cdot \mathrm{s}^{-1}$ & 2.25 & 1.77 \\
$K_{\mathrm{g}} / \times 10^{-2} \mathrm{~W} \cdot \mathrm{m}^{-1} \cdot \mathrm{K}^{-1}$ & 1.8 & 2.5 \\
\hline
\end{tabular}

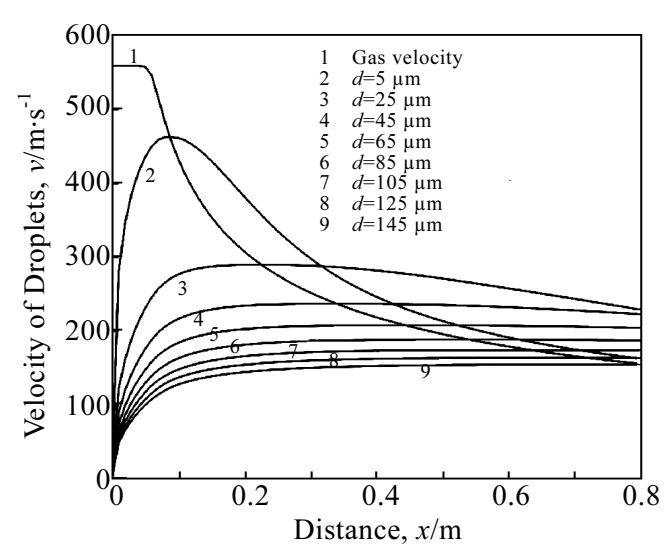

Fig. 2 Calculated velocity of different sized metallic droplets atomized by argon gas 


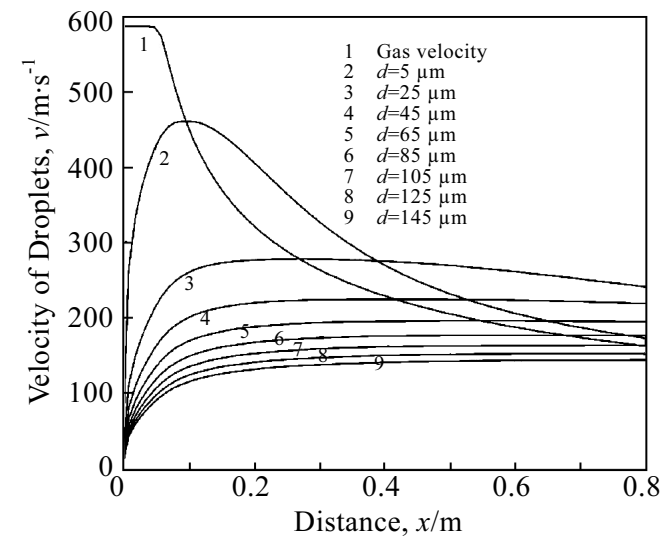

Fig. 3 Calculated velocity of different sized metallic droplets atomized by nitrogen gas

is obtained not only in shorter flight time but also at smaller flight distance. Beyond the flight distance where the maximum velocity is reached, the droplet travels faster than the atomizing gas and consequently it is decelerated. On the other hand, the diameter for the droplets larger than $85 \mu \mathrm{m}$ do not exhibit a marked deceleration because of their inertia force. Therefore, larger droplet velocities keep roughly constant after the maximum velocity, resulting in an approximately linear relation between the flight time and the flight distance except an initial period. The argon gas atomized droplets more rapidly attain their maximum velocity assuming the same droplet diameter. For example, the maximum velocity for $25 \mu \mathrm{m}$ droplets atomized by argon gas is $287 \mathrm{~m} / \mathrm{s}$ at a distance of $0.23 \mathrm{~m}$, whereas the maximum velocity for $25 \mu \mathrm{m}$ droplets atomized by nitrogen gas is $278 \mathrm{~m} / \mathrm{s}$ at a distance of $0.27 \mathrm{~m}$ from the atomizer.

Assuming that the heat transfer between spherical metallic droplets and atomizing gas flow field follows a Newtonian cooling condition, the cooling rate of droplets can be expressed as ${ }^{[20,21]}$ :

$$
\frac{\mathrm{d} T_{\mathrm{d}}}{\mathrm{d} t}=-\frac{6 h\left(T_{\mathrm{d}}-T_{\mathrm{g}}\right)}{\rho_{\mathrm{d}} C_{1} d}
$$

where $T_{\mathrm{d}}$ and $T_{\mathrm{g}}$ are droplets temperature and gas temperature, respectively, $\rho_{\mathrm{d}}$ is the density of droplet, $C_{\mathrm{t}}$ is the specific heat of droplet, $d$ is the droplet diameter, $t$ is the time and $h$ the heat transfer coefficient. According to Szekely model ${ }^{[22]}$, the heat transfer coefficient $h$ can be expressed as:

$$
h=\frac{K_{\mathrm{g}}}{d}(2.0+0.6 \sqrt{R e} \cdot \sqrt[3]{P r})
$$

where $K_{\mathrm{g}}$ is the heat transfer coefficient of gas, $d$ is the droplet diameter, $R e$ is the Reynolds number, and $\operatorname{Pr}$ is the Prandtl number which is given by ${ }^{[23]}$ :

$$
\operatorname{Pr}=\frac{C_{\mathrm{pg}} \mu_{\mathrm{g}}}{K_{\mathrm{g}}}
$$

where $C_{\mathrm{pg}}, \mu_{\mathrm{g}}$ and $K_{\mathrm{g}}$ are the gas specific heat, the gas dynamic viscosity and the gas thermal conductivity, respectively. $|u-v|$ is the relative gas-droplet velocity which can be expressed as ${ }^{[23]}$ :

$$
|u-v| \approx u_{0}\left(\frac{T_{\mathrm{d}}-T_{\mathrm{g}}}{T_{\mathrm{i}}}\right)^{2}
$$

where $\mu_{0}$ is the initial axial gas velocity at the nozzle exit, being $560 \mathrm{~m} \cdot \mathrm{s}^{-1}$ for argon gas and $580 \mathrm{~m} \cdot \mathrm{s}^{-}$for nitrogen gas. $T_{\mathrm{d}}, T_{\mathrm{g}}$ and $T_{\mathrm{i}}$ are the droplets temperature, gas temperature and the initial melting temperature $\left(T_{\mathrm{i}}=1573 \mathrm{~K}\right)$, respectively. So the initial gas-droplet relative velocity is $390 \mathrm{~m} \cdot \mathrm{s}^{-1}$ in the argon gas environment and is $404 \mathrm{~m} \cdot \mathrm{s}^{-1}$ in the nitrogen gas environment. The cooling rate of droplets can be calculated by substituting the relevant parameters listed in Table 2 into Eq. (8). The relation curves of cooling rate and powder diameter are shown in Fig. 4 for two kinds of atomizing gas. The cooling rate of argon-gas atomized powders ranges between $1.0 \times 10^{5}$ and $4.24 \times 10^{6} \mathrm{~K} \cdot \mathrm{s}^{-1}$, and the cooling rate of nitrogen-gas atomized powders ranges between $1.0 \times 10^{5}$ and $4.8 \times 10^{6} \mathrm{~K} \cdot \mathrm{s}^{-1}$.

It seems that the atomizing medium has a slight influence on the cooling rate of droplets. The cooling rate depends on the droplets size. The cooling rates decease with increasing of droplets diameter. When the droplets diameter is less than $40 \mu \mathrm{m}$, the cooling rate elevates quickly with decreasing of droplets diameter. When the droplets diameter is between 40 and $80 \mu \mathrm{m}$, the cooling rate increase slowly as the droplets size decreases. When the droplets diameter is more than $80 \mu \mathrm{m}$, the cooling rate curve flattens out with increasing of droplets size.

\subsection{Dendrite morphology and segregation at different cooling rates}

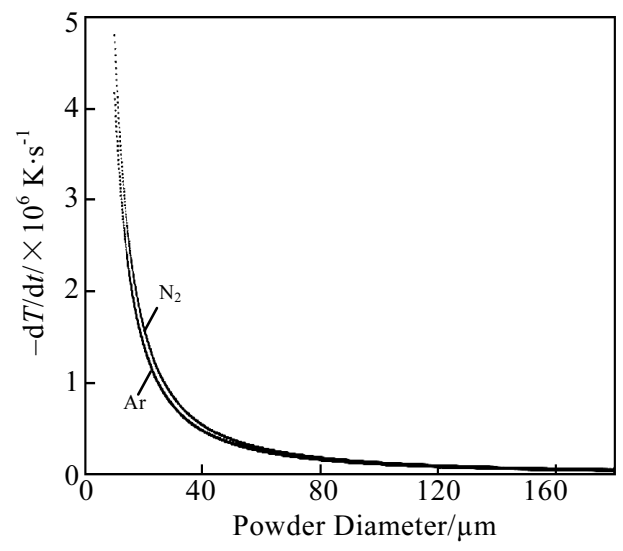

Fig. 4 Relation of cooling rate and powders diameter 
The comparison indicates that the appearance of the argon-gas atomized powders is much more regular than the nitrogen-gas atomized powders. The small powders have a better sphericity compared with the large powders. The powders morphology depends on spheroidization time and solidification time after the metallic droplets are crushed by atomizing gas. If the spheroidization time is much shorter than the solidification time, the metallic droplets are more likely to get regular shapes and show more superior surface smoothness. This may be further explained by the spheroidization time of atomized droplets which can be empirically expressed as:

$$
\tau_{\text {shp }}=\frac{3 \pi^{2} \mu_{\mathrm{L}}}{4 V \sigma} \cdot\left(\frac{1}{2}\right)^{4}\left(d_{1}^{4}-d_{2}^{4}\right)
$$

where $d_{1}$ and $d_{2}$ are the droplet diameter after spheroidization and the smallest diameter before spheroidization, respectively, $\mu_{\mathrm{L}}$ is the liquid metal viscosity, $V$ is the droplet volume and $\sigma$ is the surface tension of liquid metal. According to Eq..(12), the spheroidization time is correlated with liquid alloy viscosity, droplet volume and surface tension. If the viscosity and the surface tension are given fixed values, the small sized droplets are more easily to be spheroidized.

The variation of cooling rate with droplets diameter may has an effect on the solidification microstructure. In order to study the effect of alloy powder diameter on the solidification microstructure, the microstructural characteristics of different sized powders atomized by different gaseous media have been performed. Fig.5 shows the dendrite morphology of different sized alloy powders atomized by argon gas. The surface microstructure of the powders in the size range of $125 \sim 200 \mu \mathrm{m}$ is shown in Fig.5a. The nucleation core is on the droplet surface and a clear network of dendritic arms is visible. Secondary, tertiary and higher order arms can be developed in the cross section from the primary arms. The analysis of the surface microstructure of the droplets in the size range of $80 \sim 125 \mu \mathrm{m}$ enables us to clearly distinguish the primary dendrite arms. The location of the nucleation center is identified as the intersection of the primary dendrite arms in Fig.5b. The surface microstructure of the powders in the range of $44 \sim 80 \mu \mathrm{m}$ has the formation of a mixed microstructure composed of dendrite structure and cellular structure, which is shown in Fig.5c. With decreasing of powder size, a large proportion of cellular structure can be observed as shown in Fig.5d. The surface microstructure analysis of argon-gas atomized powders indicates that dendrite growth takes priority over cellular structure. The surface microstructure of small particles is composed of dendrite structure and cellular structure due to higher cooling rates of small droplets.

Fig. 6 shows the microstructure characteristics of different sized alloy powders atomized by nitrogen gas. The microstructure of the powders in the size range of $125 \sim 200$

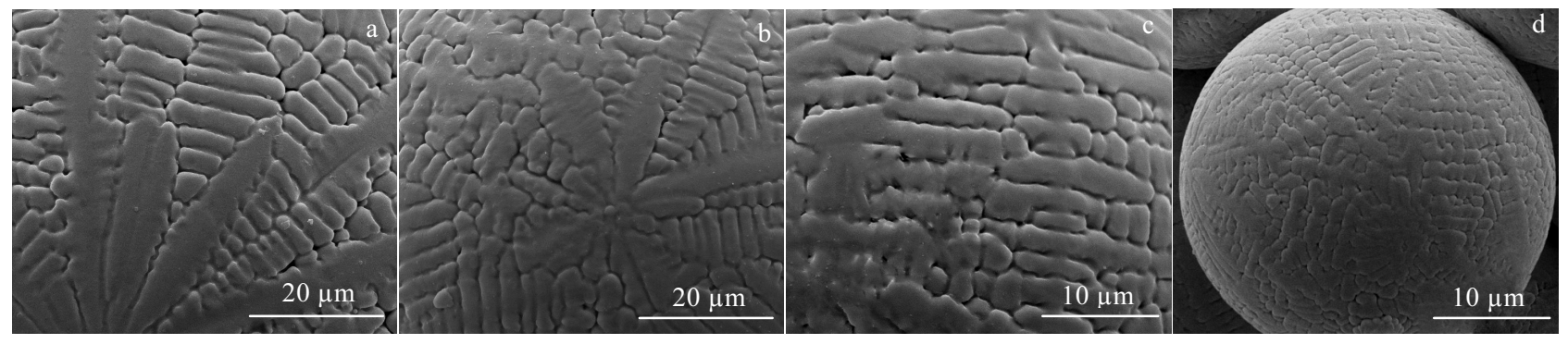

Fig. 5 Surface morphologies of argon-gas atomized powders in different size ranges: (a) $125 \sim 200 \mu \mathrm{m}$, (b) $80 \sim 125 \mu \mathrm{m}$, (c) $44 \sim 80 \mu \mathrm{m}$, and (d) $<44 \mu \mathrm{m}$

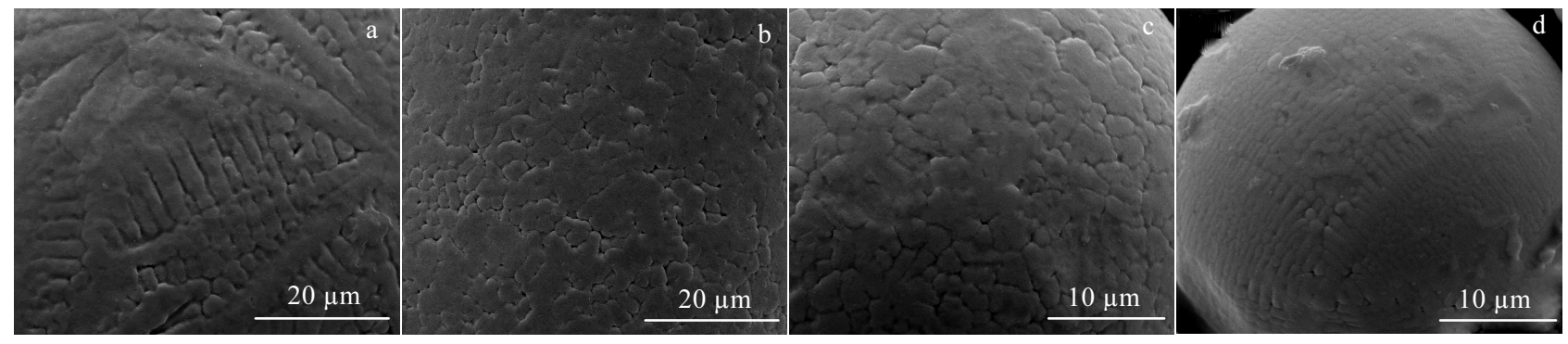

Fig. 6 Surface morphologies of nitrogen-gas atomized powders in different size ranges: (a) $125 \sim 200 \mu \mathrm{m}$, (b) $80 \sim 125 \mu \mathrm{m}$, (c) $44 \sim 80$, $\mu \mathrm{m}$, and (d) $<44 \mu \mathrm{m}$ 
$\mu \mathrm{m}$ is characterized by primary and secondary dendrites, as shown in Fig.6a. The powders in the size range of less than $125 \mu \mathrm{m}$ indicate a nearly full cellular structure. Based on the solidification nucleation theory, the nucleation critical size of the undercooled droplets relies on the competition between the driving force for the phase transformation and the energy barrier that is required for forming a new interface ${ }^{[2]}$. The energy barrier resulting in the formation of a spherical critical nucleus can be expressed as:

$$
\Delta G^{*}=\frac{16 \pi}{3}\left(\frac{\sigma^{3}}{\Delta G_{\mathrm{m}}^{2}}\right) f(\theta)
$$

where $\Delta G_{\mathrm{m}}=\Delta H_{\mathrm{m}} \cdot \Delta T / T_{\mathrm{m}}$ is the Gibbs free energy, and $\Delta T$ is the undercooling temperature of the melt alloy. The nucleation velocity is considered to depend on the undercooling degree of the melt $\Delta T$, which increases linearly with the cooling rate for a given composition. For the nickel-based alloy system in the present experimental conditions, the formation of developed dendrites of large sized particles can be accounted for by a low cooling rate. Fine cellular structure of small sized particles is related to a high cooling rate and a directional heat extraction, which in principle agrees with the calculated results of the droplets cooling rate discussed above.

The impact of atomizing gas has also been investigated by considering droplets cooled in argon gas and nitrogen gas. The microstructure analysis of the powders in the range of 125 200 $\mu \mathrm{m}$ shows that all other parameters are similar, and the dendrite morphology of atomized powders is promoted in argon-gas atomization compared with in nitrogen-gas atomization, as illustrated in Fig. 5a and Fig. 6a.
As shown in Fig.4, the droplet cooling rate variation tendencies are similar in different atomizing gas media. Based on the predicted results by the Newtonian cooling model, the nitrogen-gas atomized powders have higher cooling rates than the argon-gas atomized powders during the rapid solidification. Because the thermal conductivity of nitrogen gas is 1.4 times as much as that of argon gas, the intensity of the rapid solidification is then larger for droplets solidified in nitrogen, which is in agreement with the fact that the cellular structure of the nitrogen-gas atomized powders in the range of $<44 \mu \mathrm{m}$ is more developed than that of the argon-gas atomized powders, as illustrated in Fig. 5d and Fig. 6d.

The variation of alloy element solute concentration in different sized particles can be measured by EDS analysis. Fig. 7 and Fig. 8 show some main-alloy-element segregation characteristics in the different parts of dendrites of argongas atomized powders and nitrogen-gas atomized powders, respectively. It is obvious to notice that for the two kinds of powders, the concentration of $\mathrm{Cr}, \mathrm{Co}, \mathrm{W}, \mathrm{Ni}$ and $\mathrm{Al}$ in the dendrite axis is higher than that in the inter-dendrite region. But for $\mathrm{Ti}$, it is higher in the inter-dendrite region. The element segregation gradually weakens with the decrease of powder particle size, which is related to the powders solidification process. During the solidification process, a smaller droplet has a higher cooling rate. It is reported that increasing cooling rate enhances the solid solubility and hence decreases the micro-segregation ${ }^{[25,26]}$. The dendrite segregation is associated with cooling rate, alloy composition, powders diameter, and atomizing gas medium.
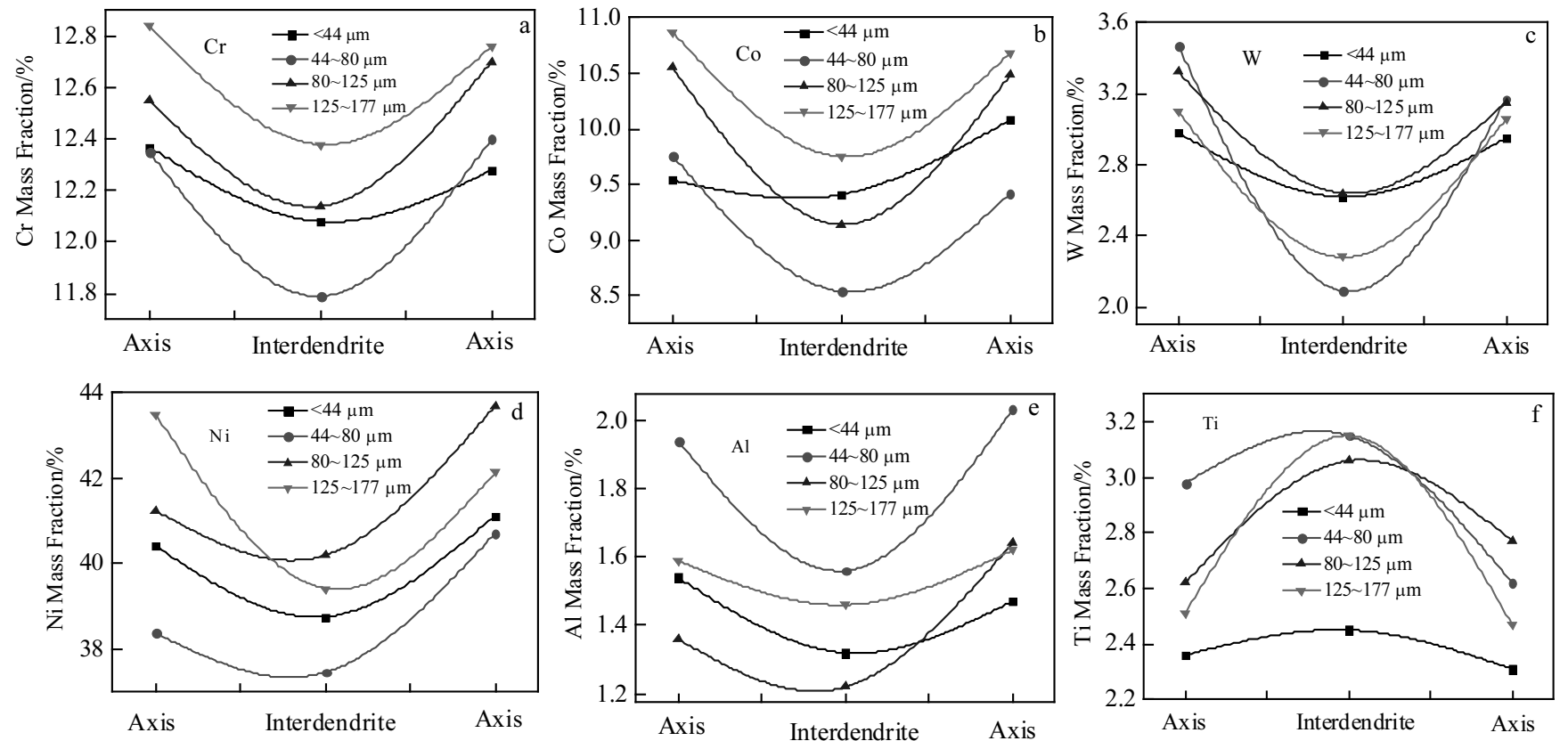

Fig.7 Element mass fraction of different sized particles atomized by Ar gas: (a) Cr, (b) Co, (c) W, (d) Ni, (e) Al, and (f) Ti 

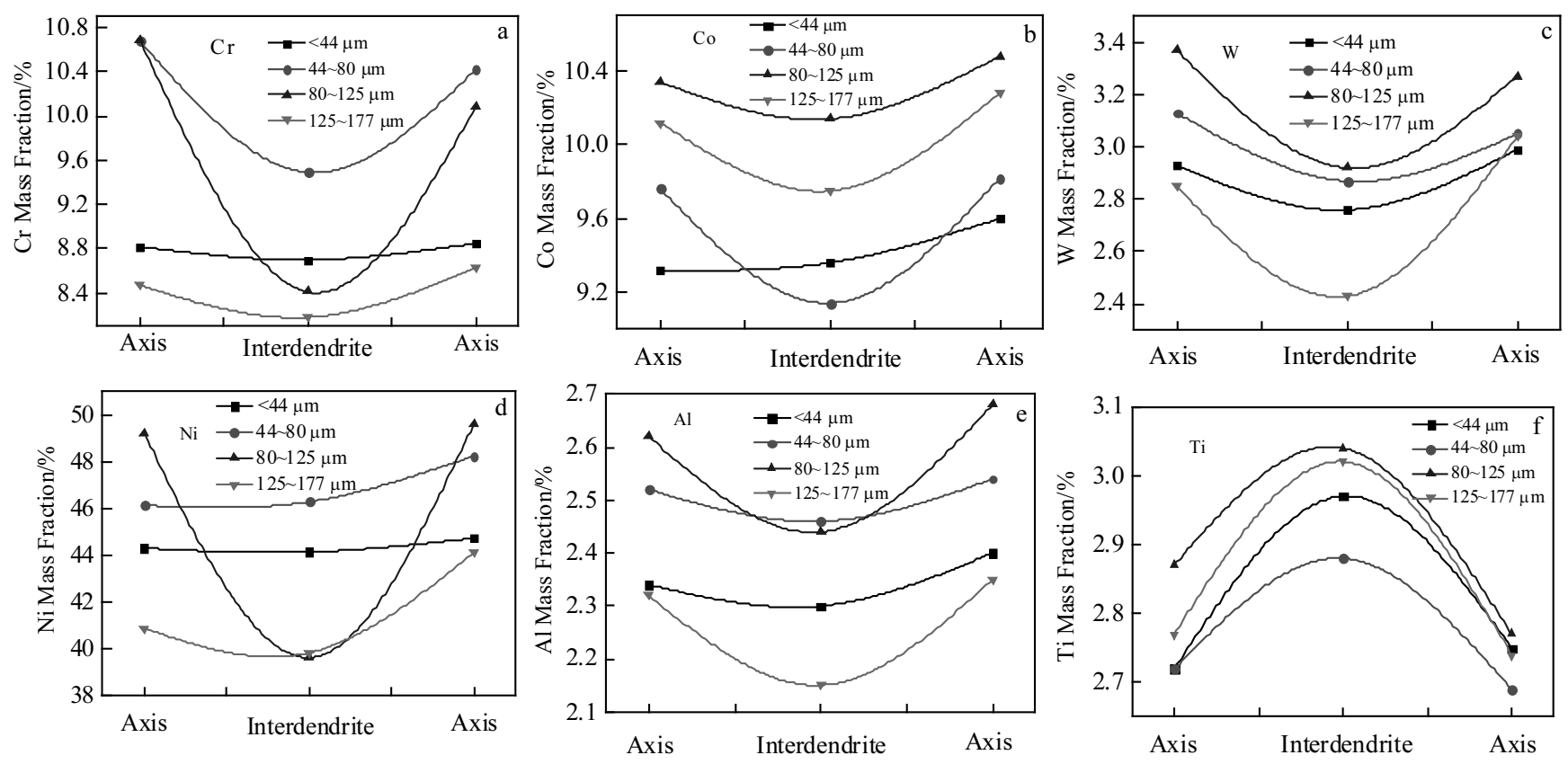

Fig. 8 Element mass fraction of different sized particles atomized by $\mathrm{N}_{2}$ gas: (a) Cr, (b) Co, (c) W, (d) Ni, (e) Al, and (f) Ti

\section{Conclusions}

1) The particle shape of Nickel-based superalloy powders tends to be more spherical in argon-gas atomization than in nitrogen-gas atomization. The microstructure of the atomized powders is affected by the atomization medium and the droplet cooling rate. For argon-gas atomized powders, the developed dendrite structure is predominant at a lower cooling rate, while a mixed microstructure composed of dendrite structure and cellular structure is obtained at a higher cooling rate. For nitrogen-gas atomized powders, the dendrite structure is predominant at a lower cooling rate, while a full cellular structure is obtained at a higher cooling rate.

2) The calculated cooling rate of argon-gas atomized droplets is in a range from $1.0 \times 10^{5} \mathrm{~K} \cdot \mathrm{s}^{-1}$ to $4.24 \times 10^{6} \mathrm{~K} \cdot \mathrm{s}^{-1}$, while that of nitrogen-gas atomized droplets from $1.0 \times 10^{5}$ $\mathrm{K} \cdot \mathrm{s}^{-1}$ to $4.8 \times 10^{6} \mathrm{~K} \cdot \mathrm{s}^{-1}$. The cooling rate increases with decreasing of particles diameter, and the cooling rates sharply increase when the droplet diameter is below $40 \mu \mathrm{m}$.

3) Some alloy elements have some micro-segregation to a certain extent. The segregation behaviors of two kinds of powders prepared by different atomization mediums follow the same variation orderliness: the elements of $\mathrm{Cr}, \mathrm{Co}, \mathrm{W}$, $\mathrm{Ni}$ and $\mathrm{Al}$ are rich in the dendrite axis, while Ti element is rich in the inter-dendrite region. The alloy-element segregation in a dendrite is associated with atomization medium and particle diameter.

\section{References}

1 Al-Jarba K A, Fuchs G E. Materials Science and Engineering $A[\mathrm{~J}], 2004,373: 255$

2 Lherbier L W, Kent W B. The International Journal of Powder Metallurgy[J], 1990, 26: 135

3 Park N K, Kim I S. Journal of Materials Processing Technology[J], 2001, 111: 99

4 Wu Q. Journal of Advanced Aerospace Materials[J], 2007, 100: 54

5 Yin Fazhang, Hu Benfu, Jin Kaisheng et al. Journal of Materials Engineering[J], 2005, 10: 52

6 Udo Fritsching, Volker Uhlenwinkel. Hybrid Gas Atomization for Powder Production[M]. German: University of Bremen Germany Press, 1993: 97

7 Yolken H Tomas, Modfin Leonard. Automated Processing of Advanced Materials[J], 1986, 32: 40

8 Kearns Martin. Materials Science and Engineering A[J], 2004, 375-377: 120

9 Umeda T, Okane T, Kurz W. Acta Materialia[J], 1996, 44: 4209

10 Freyberg A, Buchholz M, Uhlenwinkel V et al. Metallurgical and Materials Transactions[J], 2003, B34: 243

11 Levi C G, Mehrabian R. Metallurgical and Materials Transactions [J], 2002, 25: 32

12 Nayak S S, Wollgarten M, Banhart J et al. Materials Science and Engineering $A[\mathrm{~J}], 2010,527: 2370$

13 Zambon A, Badan B, Norman A F et al. Materials Science and Engineering $A[\mathrm{~J}], 1997,226: 121$

14 Lee E S, Ahn S. Acta Metallurgica Materialia[J], 1994, 42: 3238

15 Saad M A. Prentice-Hall. Compressible Fluid Flow[M]. 
Englewood Cliffs N J: Prentice Hall, 1985: 92

16

Grant P S, Cantor B, Katgerman L. Acta Metallurgica Materialia[J], 1993, 41: 3098

17 Clift R, Grace K R, Weber M E. Bubbles, Drops, and Particles [M]. New York: Academic Press,1978: 113

18 Zhang J S, Xiong B Q, Cui H. Spray Forming Rapid Solidification Technology: Principles and Applications $[\mathrm{M}]$. Beijing: Science Press, 2007: 47 (in Chinese)

19 Inoue A, Masumoyo T, Ekimoyo $\mathrm{T}$ et al. Metallurgical Transactions $A[\mathrm{~J}], 1988,19: 235$

20 Zhai Q J, Gao Y L, Guan W B et al. Materials Science and Engineering $A[\mathrm{~J}], 2006,441: 279$
21 Lee E S. Ahn S. Acta Metallurgica Materialia[J], 1994, 42: 3231

22 Szekely J, Themelis N J. Rate Phenomena in Process Metallurgy [M]. New York: Wiley-Interscience, 1971: 237

23 Lee E S, Ahn S. Acta Metallurgica Materialia[J], 1994, 42: 3231

24 Fu J W, Yang Y S. Journal of Crystal Growth[J], 2011, 322: 84

25 Suryanarayana C, Anantharaman T R. Journal of Materials Science [J], 1970, 5: 998

26 Hehmann F, Sommer F, Predel B. Material Science and Engineering $A[\mathrm{~J}], 1990,125: 242$

\title{
雾化气体和冷却速率对镍基合金粉末凝固特性的影响
}

\author{
方鹏均 ${ }^{1}$, 徐 轶 ${ }^{1,2}$, 黎兴刚 ${ }^{3}$, 陈 亚 $^{4}$ \\ (1. 西南交通大学 材料科学与工程学院, 四川 成都 610031) \\ (2. 不来梅大学 材料技术研究所, 不来梅 德国 28359) \\ (3. 北京有色金属研究总院 国家有色金属复合材料工程技术研究中心 北京 100088)
}

(4. 西南交通大学 体育科学研究所, 四川 成都 610031)

摘 要: 分别采用氩气和氮气作为雾化介质, 制备得到镍基合金粉末。利用扫描电镜分析 2 种气体雾化的粉末表面形貌及凝固组织特征。 基于牛顿冷却模型, 对雾化粉末冷却速度和飞行速度进行数值计算。结果表明: 冷却速率决定了凝固微观组织形貌。在较低的冷却速率 下， 2 种气体雾化粉末均呈现发达的枝晶组织，而随着冷却速率的增大，氩气雾化粉末呈现欠发达的枝晶和胞晶混合组织。而氮气雾化 粉末仅呈现胞晶组织。不同粒径氩气雾化粉末冷却速率在 $1.0 \times 10^{5} \sim 4.24 \times 10^{6} \mathrm{~K} \cdot \mathrm{s}^{-1}$, 氮气雾化粉末在 $1.0 \times 10^{5} \sim 4.8 \times 10^{6} \mathrm{~K} \cdot \mathrm{s}^{-1}$ 。冷却速率随粉 末直径增加而减小。在快速凝固过程中, $\mathrm{Cr}, \mathrm{Co}, \mathrm{W}, \mathrm{Ni}$ 和 $\mathrm{Al}$ 等元素仍沿晶轴偏析, 而 Ti元素沿晶间偏析。

关键词：镍基合金粉末；气体雾化；凝固组织；冷却速率

作者简介: 方鹏均, 男, 1990 年生, 硕士, 西南交通大学材料科学与工程学院, 四川 成都 610031, 电话: 028-87600782, E-mail: fangpegnjun@163.com 